\title{
Using PROMs to guide patients and practitioners through the head and neck cancer journey
}

This article was published in the following Dove Press journal:

Patient Related Outcome Measures

8 November 2017

Number of times this article has been viewed

\author{
Simon N Rogers 1,2 \\ Brittany Barber ${ }^{3}$ \\ 'Evidence-Based Practice Research \\ Centre (EPRC), Faculty of Health \\ and Social Care, Edge Hill University, \\ Ormskirk, UK; ${ }^{2}$ Regional Maxillofacial \\ Unit, University Hospital Aintree, \\ Liverpool, UK; ${ }^{3} \mathrm{Head}$ and Neck \\ Department, Icahn School of Medicine \\ at Mount Sinai (MSSM), New York, \\ NY, USA
}

\begin{abstract}
The measurement of patient-reported outcome measures (PROMs) following head and neck cancer (HNC) has the capacity to substantially enhance the care of patients and their care-givers following the diagnosis and treatment of HNC. Literature concerning PROMs has increased exponentially in the past 2 decades, producing a vast array of data upon which the multidisciplinary team can reflect. For this review, "Handle On QOL" has been used as a source of references to illustrate the points raised. PROMs are contextualized by considering the clinically-distinct key stages that cancer patients endure: diagnosis, treatment, acute toxicity, early recovery, late effects, recurrence, and palliation. The PROMs are considered in six main categories: 1) those addressing cornucopia of issues not specific to cancer; 2) those addressing issues common to all cancers; 3) questionnaires with items specific to HNC; 4) questionnaires that focus on a particular aspect of head and neck function; 5) those measuring psychological concerns, such as depression, anxiety, or self-esteem; and 6) item prompt lists. Potential benefits of PROMs in clinical practice are discussed, as are barriers to use. The way forward in integrating PROMs into routine HNC care is discussed with an emphasis on information technology.
\end{abstract}

Keywords: patient reported outcomes, head and neck cancer, quality of life, multi-professional

\section{What is head and neck cancer (HNC)?}

HNCs are neoplasms of the upper aerodigestive tract and ascribed the International Classification of Diseases codes 140-149 and 160-1. ${ }^{1}$ This includes tumor sites involving the oral cavity, oropharynx, larynx, hypopharynx, paranasal sinuses, and salivary glands. HNC surgeons and oncologists, through multi-professional collaboration, are also involved in the treatment of thyroid and skull base lesions, as well as extensive facial neoplasms. Over $90 \%$ of HNCs are squamous cell carcinomas (SCC), though other non-epithelial pathologies are present, such as lymphoma, melanoma, sarcoma, and odontogenic tumors. ${ }^{2,3}$

In North America and Europe, HNCs account for 5\%-10\% of cancers; however, there is significant geographical variation spanning incidence and anatomic site worldwide. For example, in high-risk countries such as India, Sri Lanka, or Bangladesh, oral cavity SCC is the most common cancer in men and the third most common cancer in women. ${ }^{4}$ Yet, in the United States, head and neck SCC is the eighth most common cancer in men with $\sim 53,600$ patients diagnosed annually. ${ }^{5}$ This can be attributed to differences in exposure to causative carcinogenic agents globally, which contributes to development of nearly $80 \%$ of all HNSCC diagnosed worldwide. ${ }^{6}$ Causative etiologies include traditional carcinogens such as smoking, alcohol, and betel nuts. However,
Correspondence: Simon N Rogers Regional Maxillofacial Unit, Aintree University Hospital, Lower Lane, Liverpool, L9 IAE, UK

Email snrogers.aintree@gmail.com 
more recently, the human papillomavirus (HPV) has been responsible for an epidemic-like increase in the incidence of oropharyngeal SCC worldwide. ${ }^{7,8}$

\section{What treatments are involved?}

The treatment of HNC varies by site and stage of disease. ${ }^{9}$ In early cancers, single modality treatments are employed, including surgery or radiotherapy. In advanced disease, the treatment typically involves multimodality options, including surgery followed by radiotherapy, surgery, and chemoradiotherapy (CRT). Radical treatment can often be necessary to serve the main objective of survival in traditionally aggressive disease. In this type of disease, recurrence is not uncommon and often occurs within 18 months. In those who develop recurrent disease, median overall survival with current CRT regimens is $6-8$ months. ${ }^{10,11}$

Surgical ablative options include neck dissection for cervical metastasis and open, laser-, or robotic-assisted tumor extirpation. Reconstruction using local, regional, or free tissue transfer is often employed to restore function and address osteoradionecrosis in irradiated patients. ${ }^{12,13}$ Conventional external-beam radiotherapy involves 65-70 Gy over 35 treatments, yet this regimen continues to undergo refinement for HPV-related tumors. In addition, advances in intensity-modulated radiation therapy have portended reduced damage to adjacent structures, further refining the area and dose of radiation. ${ }^{14,15}$ Chemotherapy regimens are usually platinum-based, but can include taxanes and 5-fluorouracil in some institutions. Various relatively new agents have emerged targeting inhibition of the epidermal growth factor receptor, such as cetuximab, or other aspects of the innate immune system, such as ipilimumab or nivolumab. In recurrent or metastatic $\mathrm{HNC}$, these human monoclonal antibody therapies have shown promising results as checkpoint inhibitors, activating inhibited $T$ cells to attack cancer cells. ${ }^{16}$ Several of these agents are currently in trials for these advanced indications (ClinicalTrials.gov, NCT02252042). ${ }^{17}$

While often necessary to achieve the best chance of survival, the aforementioned treatments herald both shortterm (acute treatment-related toxicity) and long-term (late) adverse effects. These effects can have profound detrimental consequences on short- and long-term quality of life and well-being. ${ }^{18,19}$

\section{How does this affect patients and care-givers?}

Diagnosis and treatment of $\mathrm{HNC}$ impacts patients in physical, social, psychological, emotional, and spiritual domains.
The main areas affected are shown in Table 1. Each area can be targeted in an endeavor to improve health-related quality of life. For example, physical concerns may include chewing, eating, dry mouth (xerostomia), fatigue, or appearance. Psychological concerns may include depression or anxiety. Social concerns may include finance and employment. ${ }^{20,21}$ Poor function can portend poor HRQOL and distress. ${ }^{22}$ Previous studies have suggested that certain patient demographics are associated with poorer recovery in the domains previously specified. For example, patients scoring above 10 on the Beck Depression Inventory failed to regain normal functioning by 12 months post-diagnosis across four different HNC-specific physical domains, including speech, eating, facial esthetics, and social disruption. ${ }^{23}$ Other demographics demonstrated to impact HRQOL are marital status, ${ }^{24}$ employment status, ${ }^{25}$ educational level, ${ }^{26}$ socioeconomic status, ${ }^{27}$ and degree of comorbidity. ${ }^{28}$ The diverse nature of the individual response is signified by the abundance of published articles reporting on HRQOL following HNC. ${ }^{29}$ This complexity is reflected in the numerous abstracts identified on "Handle on QOL", a searchable web-based repository of papers that have reported QOL in HNC using questionnaires as patient-reported outcome measures (PROMs). ${ }^{29}$

\section{Who comprises the multidisciplinary team?}

In order to address the varied needs of patients and their caregivers, $\mathrm{HNC}$ care is delivered in a multidisciplinary setting. Although the composition of the team varies between units and institutions, members often include a surgical oncologist, nursing staff or clinical nurse specialist, social worker, speech and language pathologist, physiotherapist, occupational therapist, psychologist or psychiatrist, dentist, oral rehabilitation specialist, audiologist, and chaplain.

In its most ideal form, treatment of HNC (regardless of type) is delivered in tertiary cancer centers. In these

Table I Areas most commonly affected by HNC and its treatment

\begin{tabular}{ll}
\hline Carer & Coping \\
Dental status & Disfigurement \\
Emotion & Fatigue \\
Fear of recurrence & Financial/work \\
Information & Lymphedema \\
Nutrition & Oral rehabilitation \\
Pain & Personality \\
Self-esteem & Sociodemographic \\
Speech & Swallowing \\
Shoulder & Trismus \\
Xerostomia & Unknown \\
\hline
\end{tabular}

Abbreviation: HNC, head and neck cancer. 
institutions, there is a focus on expert, guideline-enforced care with defined roles across the multidisciplinary team. However, the consequences of HNC can develop and exist remote from the time of treatment, and as such, centralization of $\mathrm{HNC}$ care is necessary both to ensure close links between HNC survivors and multidisciplinary team, and between the team and locality from which the patient was referred. In the locality, the role of a clinical nurse, family physician, and dentist are very important to ensure ongoing monitoring and care of late effects of treatment, and possible referral to a tertiary care center for further management. This communication can be challenging, and as such, advances in integrating and digitalizing PROMs into routine clinical care and electronic patient records may portend improved multidisciplinary correspondence..$^{30}$

\section{What PROMs are commonly used?}

Over the past 3 decades or more, PROMs have been established as tools to illustrate patient concerns and perspective. PROMs are a key component of evaluating treatment-related effects in both cohort studies and clinical trials. They play a role in alluding to HRQOL outcomes and identifying differences in patient experience between treatment protocols. PROMs can be used as screening tools to identify specific problem issues and facilitate targeted interventions. ${ }^{31}$

PROMs are commonly utilized in a self-report questionnaire format, which is predominantly quantitative, to facilitate a defined threshold for intervention. However, qualitative approaches, including open and semi-structured interviews, have also become useful, particularly with respect to the psychological domain of HRQOL. Qualitative methodology also provides depth to our understanding and can assist in the interpretation of correlations found in questionnaire data. In addition, this information can be used to improve the information patients receive and enhance patient information leaflets and consent. ${ }^{32,33}$ Personal reflections presented from the patient perspective can have a profound impact on our appreciation of the holistic impact of HNC.

Given the complex anatomy of the head and neck, a vast array of PROMs exist to elucidate HNC symptomatology. ${ }^{34}$ "Handle on QOL" gives reference to over 250 different questionnaires. The PROMs can be considered in six main categories: 1) those addressing cornucopia of issues not specific to cancer; 2) those addressing issues common to all cancers; 3) questionnaires with items specific to $\mathrm{HNC}$; 4) questionnaires that focus on a particular aspect of head and neck function; 5) those measuring psychological concerns, such as depression, anxiety, or self-esteem; and 6) item prompt lists, such as the Patient Concerns Inventory (PCI).
Given the wide range of issues possibly affected by HNC (Table 2), it is clear that no one questionnaire has the ability to cover all aspects. Each PRO has its own unique features and merits, and there is no gold standard. The situation is further complicated as there is no consensus on the standard definition for "quality of life" or HRQOL. The measurement of HRQOL varies between institutions, ${ }^{35,36}$ and this makes assessment of large datasets difficult. However, the "quality of life" agenda has been refined with the focus on HRQOL, with most assessments concentrating on the physical, psychological, and social aspects of $\mathrm{HNC}$ and their impacts. "Handle on QOL"

Table 2 Domains housing symptoms experienced during diagnosis and treatment by HNC patients as based on the Patient Concerns Inventory item prompt list

\begin{tabular}{|l|l|}
\hline $\begin{array}{l}\text { Physical and functional } \\
\text { well-being }\end{array}$ & Treatment-related \\
\hline Activity & Cancer treatment \\
\hline Appetite & Regret about treatment \\
\hline Bowel habit & PEG tube \\
\hline Breathing & Wound healing \\
\hline Chewing/eating & \\
\hline Coughing & Social care and social well-being \\
\hline Dental health/teeth & Carer \\
\hline Dry mouth & Dependents/children \\
\hline Energy levels & Financial benefits \\
\hline Fatigue/tiredness & Home care/district nurse \\
\hline Hearing & Lifestyle issues (smoking/alcohol) \\
\hline Indigestion & Recreation \\
\hline Mobility & Relationships \\
\hline Mouth opening & Speech/voice/being understood \\
\hline Mucous & Support for my family \\
\hline Nausea & \\
\hline Pain in the head and neck & Psychological, emotional and \\
& spiritual well-being \\
\hline Pain elsewhere & Appearance \\
\hline Regurgitation & Angry \\
\hline Salivation & Anxiety \\
\hline Shoulder & Coping \\
\hline Sleeping & Depression \\
\hline Smell & Fear of the cancer coming back \\
\hline Sore mouth & Fear of adverse events \\
\hline Swallowing & Intimacy \\
\hline Swelling & Memory \\
\hline Taste & Mood \\
\hline Vomiting/sickness & Self-esteem \\
\hline Weight & Sexuality \\
\hline & Spitual/religious aspects \\
\hline & Abrity and temperament \\
\hline
\end{tabular}

Abbreviations: HNC, head and neck cancer; PEG, percutaneous endoscopic gastrostomy tube. 
provides access to over 150 papers on questionnaire development and validation. ${ }^{29}$ While most PROMs can be categorized as stated previously, it can often be more clinically intuitive to professionals and patients to classify them according to the key stages encountered by the patient. While the length and adversity of each stage may vary between patients, the patients present with similar concerns at each stage that can be quantified and monitored. Questionnaires relevant to the described key stages are demonstrated in Table 3.

As treatments for HNC change over time, existing questionnaires require adjustment to ensure accurate assessment. An example of this is the European Organization for Research and Treatment of Cancer (EORTC) QOL Module for HNC (QLQ-H\&N43). ${ }^{37}$ This was felt advisable given the deficits in the original EORTC HNC module in relation to targeted and/or multimodal therapy. ${ }^{38}$

\section{What are the key periods in the patient journey?}

HNC patients can experience symptoms in clinically distinct key stages, outlined as diagnosis, treatment, acute toxicity, early recovery, late effects, recurrence, and palliation.

\section{Diagnosis}

Disclosing a diagnosis of $\mathrm{HNC}$ to a patient requires an individually-tailored approach. Information provided to patients should be clear, concise, and specific to the individual, as each patient desires a different level of detail in explanation. ${ }^{39}$ Additionally, PROMs may assist with decision-making regarding chosen treatment path following diagnosis. Questionnaires exist to allow clinicians to assess satisfaction with information provision among HNC patients. An example of this is the Satisfaction with Cancer Information profile, a validated measure of satisfaction with information about treatment, which is responsive to change. ${ }^{40}$

The Patient Concerns Inventory (Diagnosis) (PCI-D) is an item prompt list intended to prompt patients to inquire about aspects of their cancer diagnosis, potential treatments, and outcomes. ${ }^{41}$ Aside from assessing patient symptomatology, it additionally provides a prompt list to prioritize items of discussion with different members of the multidisciplinary team.

The feasibility of answering the question, "What will I be like after my treatment of HNC?" has been improved with expanded, longitudinal PRO datasets. ${ }^{42,43}$ These datasets can yield further detailed subgroup analysis of HRQOL, which may in turn provide patients and practitioners with a more accurate extrapolation of long-term PROs. However, the benefit of managing patient expectations by forecasting HRQOL outcomes after treatment needs further evaluation.

\section{Treatment}

As mentioned, qualitative methods have been used to inform patients and practitioners as to the patient perspective during treatment. Examples of this may include patients' experience

Table 3 Examples of patient-reported outcomes relating to HRQOL during key stages of HNC

\begin{tabular}{|c|c|}
\hline Diagnosis & $\begin{array}{l}\text { - Patient Concerns Inventory-Diagnosis }{ }^{41} \\
\text { - Satisfaction with Cancer Information profile }(\mathrm{SCIP})^{40}\end{array}$ \\
\hline Treatment & $\begin{array}{l}\text { - Hospital Anxiety Depression Scale (HADS) })^{83} \\
\text { - Symptom Severity Scale (SSS) } \\
\text { - Visual Analog Scale (VAS) }\end{array}$ \\
\hline Acute toxicity & $\begin{array}{l}\text { - Late Effects in Normal Tissue (LENT) }{ }^{86} / \text { Subjective-Objective-Management-Analytic (SOMA) } \\
\text { - Common Terminology Criteria for Adverse Events (CTC-AE) } \\
\text { - Oral Mucositis Weekly Questionnaire-Head and Neck Cancer (OMWQ-HN) }{ }^{88}\end{array}$ \\
\hline Early recovery & $\begin{array}{l}\text { - EuroQOL EQ-5D-5 } 5 L^{89} \\
\text { - European Organization for Research and Treatment of Cancer (EORTC) QLC-C } 30^{90} \text { and EORTC H\&N } 35^{91} \\
\text { - Functional Assessment of Cancer Therapy-Head and Neck (FACT-H\&N) }{ }^{92} \\
\text { - University of Washington Quality of Life Scale (UW-QoL v4) })^{93} \\
\text { - Patient Concerns Inventory (PCl-HN) }\end{array}$ \\
\hline Late effects & $\begin{array}{l}\text { - Oral rehabilitation: Liverpool Oral Rehabilitation Questionnaire (LORQ) }{ }^{94} \\
\text { - Trismus: Gothenburg Trismus Questionnaire (GTQ) } \\
\text { - Swallowing: Swallowing Quality of Life Questionnaire (SWAL-QOL) })^{96} \\
\text { - Xerostomia Questionnaire (XQ) }{ }^{97}\end{array}$ \\
\hline Recurrence & - Vanderbilt Head and Neck Symptom Survey (VHNSS) ${ }^{98}$ \\
\hline Palliation & $\begin{array}{l}\text { - EORTC QLQ-CI5-PAL99 } \\
\text { - Family Assessment of Treatment at the End of life (FATE) })^{100} \\
\text { - Schedule for the Evaluation of Individual Quality of Life (SEIQoL) }{ }^{76}\end{array}$ \\
\hline
\end{tabular}

Abbreviation: HRQOL, health related quality of life; HNC, head and neck cancer. 
of temporary tracheostomies after microvascular reconstruction for cancer of the head and neck, ${ }^{33}$ and patients' experiences in the early recovery phase following carbon dioxide laser removal of oral SCC. ${ }^{32}$ These approaches help to highlight predominant patient concerns to the treatment team and enhance preoperative teaching and patient resource materials.

PROMs are also used to help inform patients about differences in short- and long-term morbidity between different treatment options. This becomes pertinent when no discussion regarding the trade-off between cure and HRQOL is planned. In this situation, the compromise is weighted toward treatment that offers the best chance of cure. However, PROMs can be very useful when discussing a specific aspect of the treatment, such as the comparison of patient- and surgeon-rated morbidity following deep circumflex iliac artery and fibula free tissue transfer. ${ }^{44}$ This point can be additionally demonstrated in circumstances when survival between two treatments is very similar. For example, in early laryngeal cancer, PROMs may reflect differences in HRQOL priorities and aid in decisionmaking between primary radiotherapy and laser ablation. ${ }^{45}$

PRO assessment during treatment adds an additional patient burden at a challenging time. Hence, to date, there are relatively few studies in this area. Some institutions are now employing the use of tablet devices and web applications to provide a more comfortable environment for repeated PRO reporting by the patient. ${ }^{46,47,48}$ This concept will be discussed in more detail later in 'The way forward' section.

\section{Acute toxicity}

Historically, acute toxicity has not been the focus of HRQOL assessment, given its relatively brief duration in the patient journey. However, PROMs in this key stage of HNC care can impact further coping and rehabilitation. In 1995, the EORTC and Radiation Therapy Oncology Group developed the Late Effects Normal Tissue Task Force - Subjective, Objective, Management, Analytic (LENT-SOMA) scale (31-item scale assessing mucosal integrity, management of ulceration, skin (edema, alopecia, pigmentation, necrosis, and fibrosis), salivary glands, trismus, and dentition. ${ }^{49}$ In 2010, the National Cancer Institute published the Common Terminology Criteria for Adverse Events, which incorporated the LENT-SOMA items with early and late effects of head and neck radiotherapy. ${ }^{50}$ A relationship was observed between toxicity as measured by the LENT-SOMA scale and HRQOL as measured by EORTC QLQ-C30 and QLQ-H\&N35. ${ }^{51}$ Similar findings have been reported when using the observer-based toxicity scoring system published by the Danish Head and Neck Cancer study group. ${ }^{19}$ While PROMs were not included in these assessment tools, the aforementioned study findings would support a need for their inclusion in future versions. ${ }^{19}$

Previous studies have demonstrated that real-time collection of PRO data can be used to monitor adverse events during radiotherapy or concurrent CRT treatments. When completed consistently throughout treatment, congruent early intervention can be employed to mitigate the toxicity of treatment. ${ }^{49}$

\section{Early recovery}

PROMs completed soon after the completion of treatment can help to screen patients for dysfunctional symptoms that may delay or inhibit rehabilitation, temporarily or permanently. The degree of dysfunction can vary between patients and may not necessarily equate to the objective assessment. For example, some patients may report minimal dysphagia symptoms, yet display silent aspiration on imaging studies. Other patients may report significant cosmetic concerns, which may not be readily evident during casual interaction. Yet, combining objective outcome measures with PRO may allow for targeted interventions in the early stages of prospective studies or clinical trials. The majority of PROM papers address the "early recovery" phase following HNC. While there is no precise definition of "early", it would be reasonable to consider this as from 3 months post-treatment completion to up to 5 years. There are several review articles which summarize the choice and selection of PRO available..$^{35,36,52-55}$

Common issues during this key stage are fear of recurrence, dental health/teeth, taste, salivation, chewing, swallowing, mouth opening, fatigue, sleeping, speech, and pain. ${ }^{56,57}$ Not unexpectedly, given the complexity of the head and neck region, a multitude of these concerns arise during the clinic interview. The PCI-HN is an HNC-specific prompt list to help enable patients to raise concerns in a prioritized manner. ${ }^{58}$ The PCI has 57 items and can be used alongside standard HNC cancer-specific HRQOL questionnaires. ${ }^{59}$ This combination of PROMs is useful as it allows a measure of dysfunction as well as an indication of the degree to which it concerns the patient. Therein, by reflecting on patient-prioritized issues within the PCI or other PROMs, it is possible to refine treatments to target PROMs dysfunction without diminishing cure rates. For example, priorities in patients treated by primary surgery for oral and oropharyngeal cancer are chewing and swallowing. ${ }^{60}$

PROMs can give an indication of cost utility, qualityadjusted life years, and a suggestion of health care costs when combined with direct and indirect cost assessments. A questionnaire of established repute in this context has been developed by the EuroQol Group (EQ5D-5L), and 
this PRO is a generic questionnaire suitable for other health states and $\mathrm{HNC}{ }^{61}$

\section{Late effects}

Late effects often present as delayed dysfunction as a result of previous cancer treatment. These effects often include xerostomia, dental decay, dysphagia, pathologic fractures, soft tissue fibrosis, and osteoradionecrosis, and often arise many months or years post-treatment. ${ }^{62,63}$

Mandibular osteoradionecrosis results from compromised blood supply to the mandible as a result of radiotherapy. Osteoradionecrosis can be painful and cause difficulties maintaining a normal diet and weight, imparting significant detrimental effects on HRQOL. ${ }^{64}$ Free tissue transfer with an osteocutaneous free flap can replace the necrotic bone and allow for chewing rehabilitation; however, only a modest improvement in HRQOL is observed postoperatively. ${ }^{65}$ While a reduction in pain is offered by free tissue transfer, other persistent late effects from radiotherapy such as trismus, xerostomia, and speech and swallowing difficulties hinder further improvements in HRQOL.

The reduction in saliva, the direct damage to teeth structure by radiotherapy, and the reduced turnover of tissue related to the periodontal membrane leads to radiationinduced carries, periodontal disease, and earlier than expected dental extractions. The combination of reduction in salivary flow, compromised blood supply to periodontal structures, and reduced cell turnover in the periodontal membrane can cause significant dental decay in the late effect stage, often requiring complete dental extractions. Given the noted priority of odontogenic concerns in most PROMs, ongoing support and monitoring of the remaining dentation is essential. For a select group of patients, placement of osseointegrated implants can positively impact HRQOL outcomes. ${ }^{66}$ As there is often a need to justify placement of a resource-intensive intervention such as osseointegrated implants, assessment of the potential improvement in HRQOL has previously been demonstrated on the Liverpool Oral Rehabilitation Questionnaire. $^{67}$

Dysphagia and chewing difficulties remain a persistent hindrance in the late effects stage. This is thought to be due to progressive fibrosis of the pharyngeal constrictor muscles, larynx, and the proximal esophageal sphincter. ${ }^{68}$ Severe dysphagia may develop or progress years after head and neck radiotherapy and lead to gastrostomy dependence. ${ }^{63}$ Permanent gastrostomy tubes have previously been associated with the poorest PROMs through interference with family life, intimate relationships, social activities, and hobbies. ${ }^{69}$
Late effects of radiotherapy or chemotherapy are not easy to predict and can be dose-dependent or pharmacologically specific. Progressive cognitive dysfunction can be a ubiquitously reported PRO, particularly in elderly cancer patients. However, patients undergoing CRT for cancer of the nasopharynx are particularly at risk of this outcome. ${ }^{70}$

\section{Recurrence}

Recurrent and/or unresectable HNC is a challenging scenario. Treatment is of limited benefit, and often associated with significant toxicity. Thus, projected HRQOL as reported on PROMs should be prioritized in the choice of treatment. ${ }^{71}$ HRQOL of patients receiving platinum-based chemotherapy plus cetuximab first line for recurrent and/or metastatic $\mathrm{HNC}$ has been shown to be acceptable. ${ }^{72}$ However, of interest is the effect of administration of immune checkpoint inhibitors on HRQOL, such as nivolumab, in patients with platinum-refractory, recurrent HNC. A previous study has demonstrated better PROMs and longer overall survival with administration of nivolumab when compared to that of conventional agents. ${ }^{16}$

\section{Palliation}

While the preceding key stages in the HNC journey can be prolonged, time spent in the palliative stage is often limited to several months. During this time, treatments are provided in short intervals and attempts are made to considerably reduce treatment toxicity. ${ }^{73}$

A scope for PROM-dictated palliative treatments for HNC exists, pertaining to death outside of the hospital and palliative care team involvement. ${ }^{74}$ For example, the use of the Family Assessment of Treatment at the End of life may improve both care-giver and patient support during this stage. ${ }^{75}$ The importance of care-giver support in HNC cannot be underestimated. ${ }^{75}$ As both physical and psychological concerns can change rapidly during this phase, PROMs using patient-generated indices have merit. An example of this exists in the Schedule for the Evaluation of Individual Quality of Life. ${ }^{76}$ These are more time consuming to complete and uniquely derived by individuals, hence are not frequently reported in the $\mathrm{HNC}$ literature.

\section{Potential benefits of using PROMS to guide patients and professionals}

Using PROMs to inform and guide both patients and professionals in clinical practice can confer a myriad of benefits. The main benefits, as discussed in the previous sections, are in providing patient information, predicting HRQOL, 
enhancing screening, targeting interventions, and improving communication between the patient and multidisciplinary team within the cancer center and the locality.

More recently, authors have directed attention toward creating PROMs that are more clinically applicable and focused on well-defined patient groups and treatments. This data will allow for more informed shared decision-making strategies amongst the multidisciplinary team. ${ }^{77}$

\section{Barriers to using PROMS to guide patients and professionals about the patients}

Predominant reasons cited by head and neck consultants for not using HRQOL questionnaires included lack of resources and unproven value. ${ }^{78}$ Authors have previously identified a misconception amongst clinicians affirming that PROMs are a research tool only rather than an adjunct to providing patient care and education. ${ }^{79}$ In addition, including these assessments can introduce both a time and paper burden to clinical practice. However, as discussed in the next section, the use of information technology can reduce burden and may, in fact, improve clinical efficiency while providing patient care and education. As such, future studies must capitalize on building an evidence base for the contribution of PROMs to the care of the HNC patient.

\section{The way forward}

\section{The digital revolution}

The utility of PROMs moving forward will be enhanced by the inevitable digital revolution of the health care sector. PROMs provided by web applications on smartphones and tablets will allow remote and out-of-hospital reporting. By the same token, alerting the treatment team to significant or severe concerns will be facilitated through the use of web applications. Contemporaneous collection of data by practitioners will allow for efficient review and targeted interventions across a range of physical, emotional, social, spiritual, and family domains. The efficiency provided by PROMs in the care of HNC patients may have implications for reduction in overall morbidity and subsequent health care costs. PROMs will allow for earlier identification of struggling patient, and provide an opportunity for targeted intervention prior to physical or psychological deterioration. The integration of PROMs into routine care may also reduce overall health care costs. There are several examples of how PROMs are being framed within the context of digitized care in HNC, such as the dashboard of "Kaiku", ${ }^{48}$ electronic PRO and toxicities, ${ }^{49}$ electronic QOL assessment using tablet devices during and after treatment, ${ }^{47}$ the eHealth application "OncoKompas", ${ }^{80}$ and the PCI. ${ }^{81}$

\section{The enhanced clinical interaction}

The combination of using established HRQOL questionnaires and an item prompt list like the PCI opens up the opportunity for enhanced consultations. ${ }^{59}$ This approach recognizes the limitations inherent in HNC HRQOL questionnaires with respect to a large scope of issues, but acknowledges the value of validated tools to indicate those patients with worse outcomes. It also realizes the value of allowing patients to identify a list of prioritized issues to discuss during the clinical interaction. These two approaches are efficient enough to be integrated into routine clinical care, with all the advantages this confers. ${ }^{82}$ Patients find the completion of such tools relatively uncomplicated, and by using a digital platform it is possible to build in ComputerAdaptive Tests which allow for the answer to one item of a questionnaire to lead to another specified question. Thus, the questionnaire battery can be individualized to the patient through adaptation of the questions asked, thereby optimizing measurement precision and minimizing questionnaire burden. ${ }^{82}$

\section{Conclusion}

PROMs present an opportunity for enhanced discussion between the clinician and patient throughout the HNC journey. The value of PROMs has evolved beyond the research context, and now lies in providing patient information, predicting HRQOL, enhancing screening, targeting interventions, and improving communication between the patient and multidisciplinary team. The enhanced clinical interaction delivered by the use of PROMs in diagnosis and treatment can allow for tailored rehabilitation and further refinement of future, rapidly evolving treatment regimens. Digitalization of PROMs may provide congruently prompt real-time patient feedback to better deliver patient care. This review was conducted in accordance with the principles of the Declaration of Helsinki.

\section{Disclosure}

The authors report no conflicts of interest in this work.

\section{References}

1. ICD-10 Version: 2016. International Statistical Classification of Diseases and Related Health Problems 10th Revision. Available from: http://www.who.int/classifications/icd/en/.

2. Adeyemi B, Adelkunle L, Kolude B, Akang E, Lawoyin J. Head and neck cancer - a clinicopathological study in a tertiary care centre. J Natl Med Assoc. 2008;100(6):690-697. 
3. Ologe F, Adeniji KA, Segun-Busari S. Clinicopathological study of head and neck cancers in Ilorin, Nigeria. Trop Doct. 2005;35(1):2-4.

4. Warnakulasuriya S. Global epidemiology of oral and oropharyngeal cancer. Oral Oncol. 2009;45(4-5):309-316.

5. Siegel R, Naishadham D, Jemal A. Cancer statistics, 2013. CA Cancer J Clin. 2013;63(1):11-30.

6. Vigneswaran N, Williams M. Epidemiologic trends in head and neck cancer and aids in diagnosis. Oral Maxillofac Surg Clin North Am. 2014;26(2):123-141.

7. Sturgis EM, Cinciripini PM. Trends in head and neck cancer incidence in relation to smoking prevalence: an emerging epidemic of human papillomavirus-associated cancers? Cancer. 2007;110(7):1429-1435.

8. Ramqvist T, Dalianis T. Oropharyngeal cancer epidemic and human papillomavirus. Emerg Infect Dis. 2010;16(11):1671-1677.

9. Rogers SN, Semple C, Babb M, Humphris G. Quality of life considerations in head and neck cancer: United Kingdom National Multidisciplinary Guidelines. J Laryngol Otol. 2016;130(S2):S49-S52.

10. Gibson M, Li Y, Murphy B, et al. Randomized phase III evaluation of cisplatin plus fluorouracil versus cisplatin plus paclitaxel in advanced head and neck cancer (E1395): an intergroup trial of the Eastern Cooperative Oncology Group. J Clin Oncol. 2005;23(15):3562-3567.

11. Colevas AD. Chemotherapy options for patients with metastatic or recurrent squamous cell carcinoma of the head and neck. J Clin Oncol. 2006;24(17):2644-2652.

12. Coskunfirat OK, Wei FC, Huang WC, Cheng MH, Yang WG, Chang YM. Microvascular free tissue transfer for treatment of osteoradionecrosis of the maxilla. Plast Reconstr Surg. 2005;115(1):54-60.

13. Buchbinder D, St Hilaire $H$. The use of free tissue transfer in advanced osteoradionecrosis of the mandible. J Oral Maxillofac Surg. 2006;64(6):961-964.

14. Bhide SA, Newbold KL, Harrington KJ, Nutting CM. Clinical evaluation of intensity-modulated radiotherapy for head and neck cancers. Br J Radiol. 2012;85(1013):487-494.

15. Wang X, Eisbruch A. IMRT for head and neck cancer: reducing xerostomia and dysphagia. J Radiat Res. 2016;57(Suppl 1):i69-i75.

16. Ferris RL, Blumenschein G Jr, Fayette J, et al. Nivolumab for recurrent squamous-cell carcinoma of the head and neck. $N$ Engl J Med. 2016;375(19):1856-1867.

17. Merck Sharp \& Dohme Corp. Pembrolizumab (MK-3475) versus standard treatment for recurrent or metastatic head and neck cancer (MK-3475-040/KEYNOTE-040)(2014). Available from: https:// clinicaltrials.gov/ct2/show/NCT02252042. NLM identifier: 02252042. Accessed October 9, 2017.

18. Langendijk JA, Doornaert P, Verdonck-de Leeuw IM, Leemans CR, Aaronson NK, Slotman BJ. Impact of late treatment-related toxicity on quality of life among patients with head and neck cancer treated with radiotherapy. J Clin Oncol. 2008;26(22):3770-3776.

19. Jensen K, Bonde Jensen A, Grau C. The relationship between observerbased toxicity scoring and patient assessed symptom severity after treatment for head and neck cancer. A correlative cross sectional study of the DAHANCA toxicity scoring system and the EORTC quality of life questionnaires. Radiother Oncol. 2006;78(3):298-305.

20. Egestad H, Nieder C. Undesirable financial effects of head and neck cancer radiotherapy during the initial treatment period. Int J Circumpolar Health. 2015;74:10-25.

21. Baxi S, Salz T, Xiao H, et al. Employment and return to work following chemoradiation in patients with HPV-related oropharyngeal cancer. Cancers of the Head \& Neck. 2016;1:1-8.

22. Ichikura K, Yamashita A, Sugimoto T, Kishimoto S, Matsushima E. Persistence of psychological distress and correlated factors among patients with head and neck cancer. Palliat Support Care. 2016;14(1):42-51.

23. Howren MB, Christensen AJ, Karnell LH, Funk GF. Health-related quality of life in head and neck cancer survivors: impact of pretreatment depressive symptoms. Health Psychol. 2010;29(1):65-71.

24. Long SA, D'Antonio LL, Robinson EB, Zimmerman G, Petti G, Chonkich G. Factors related to quality of life and functional status in 50 patients with head and neck cancer. Laryngoscope. 1996;106(9 Pt 1):1084-1088.
25. Allison PJ, Locker D, Wood-Dauphinee S, Black M, Feine JS. Correlates of health-related quality of life in upper aerodigestive tract cancer patients. Qual Life Res. 1998;7(8):713-722.

26. Duffy SA, Terrel JE, Valenstein M, Ronis D, Copeland LA, Connors M. Effect of smoking, alcohol and depression on the quality of life of head and neck cancer patients. Gen Hosp Psychiatry. 2002;24(3):140-147.

27. Vartanian JG, Carvahlo AL, Toyota J, Kowalski IS, Kowalski LP. Socioeconomic effect of and risk factors for disability in long-term survivors of head and neck cancer. Arch Otolaryngol Head Neck Surg. 2006;132(1):32-35.

28. Fang FM, Chiu HC, Kuo WR, et al. Health-related quality of life for nasopharyngeal carcinoma patients with cancer-free survival after treatment. Int J Radiat Oncol Bio Phys. 2002;53(4):959-968.

29. handle-on-qol.com [homepage on the Internet]. What is HaNDLEon-QoL? Available from: http://www.handle-on-qol.com/About.aspx. Accessed October 9, 2017.

30. Krebber AM, Leemans CR, de Bree R, et al. Stepped care targeting psychosocial distress in head and neck and lung cancer patients: a randomized clinical trial. BMC Cancer. 2012;12:173.

31. Mokkink LB, Terwee CB, Patrick DL, et al. The COSMIN checklist for assessing the methodological quality of studies on measurement properties of health status measurement instruments: an international Delphi Study. Qual Life Res. 2010;19(4):539-549.

32. Gilmartin M, Ali T, Rogers SN. Patients' experience in the early recovery phase after removal of intraoral squamous cell carcinoma with carbon dioxide laser. Br J Oral Maxillofac Surg. 2017;55(4): 388-390.

33. Rogers SN, Russell L, Lowe D. Patients' experience of temporary tracheostomy after microvascular reconstruction for cancer of the head and neck. Br J Oral Maxillofac Surg. 2017;55(1):10-16.

34. Rogers SN. Improving quality-of-life questionnaires in head and neck cancer. Expert Review of Quality of Life in Cancer Care. 2016;1(1): 61-71.

35. Vartanian JG, Rogers SN, Kowalski LP. How to evaluate and assess quality of life issues in head and neck cancer patients. Curr Opin Oncol. Epub 2017 Mar 9.

36. Pusic A, Liu JC, Chen CM, et al. A systematic review of patientreported outcome measures in head and neck cancer surgery. Otolaryngol Head Neck Surg. 2007;136(4):525-535.

37. Singer S, Araújo C, Arraras JI, et al. Measuring quality of life in patients with head and neck cancer: Update of the EORTC QLQ-H\&N Module, Phase III. Head Neck. 2015;37(9):1358-1367.

38. Singer S, Arraras JI, Baumann I, et al. Quality of life in patients with head and neck cancer receiving targeted or multimodal therapy - update of the EORTC QLQ-H\&N35, Phase I. Head Neck. 2013;35(9):1331-1338.

39. Newell R, Ziegler L, Stafford N, Lewin R. The information needs of head and neck cancer patients prior to surgery. Ann R Coll Surg Engl. 2004;86(6):407-410.

40. Llewellyn CD, Horne R, McGurk M, Weinman J. Development and preliminary validation of a new measure to assess satisfaction with information among head and neck cancer patients: the satisfaction with cancer information profile (SCIP). Head Neck. 2006;28(6):540-548.

41. Byrne MJ, Rogers SN. Service evaluation of patients' views on the Patients' Concerns Inventory (at diagnosis). Br J Oral Maxillofac Surg. 2017;55(7):714-716.

42. Rogers SN, Hogg ES, Cheung WK, Lai LK, Jassal P, Lowe D. The use of health related quality of life data to produce information sheets for patients with head and neck cancer. Ann R Coll Surg Engl. 2015;97(5):359-363.

43. Rogers SN, Hogg ES, Cheung WK, Lai LK, Jassal P, Lowe D, Kanatas A. "What will I be like" after my diagnosis of head and neck cancer? Eur Arch Otorhinolaryngol. 2015;272(9):2463-2472.

44. Rogers SN, Lakshmiah SR, Narayan B, et al. A comparison of the long-term morbidity following deep circumflex iliac and fibula free flaps for reconstruction following head and neck cancer. Plast Reconstr Surg. 2003;112(6):1517-1525; discussion 1526-1527. 
45. Stoeckli SJ, Guidicelli M, Schneider A, Huber A, Schmid S. Quality of life after treatment for early laryngeal carcinoma. Eur Arch Otorhinolaryngol. 2001;258(2):96-99.

46. Pollom EL, Wang E, Bui TT, et al. A prospective study of electronic quality of life assessment using tablet devices during and after treatment of head and neck cancers. Oral Oncol. 2015;51(12): 1132-1137.

47. Peltola MK, Lehikoinen JS, Sippola LT, Saarilahti K, Mäkitie AA. A novel digital patient-reported outcome platform for head and neck oncology patients-a pilot study. Clin Med Insights Ear Nose Throat. 2016:9:1-6.

48. Niska JR, Halyard MY, Tan AD, Atherton PJ, Patel SH, Sloan JA. Electronic patient-reported outcomes and toxicities during radiotherapy for head-and-neck cancer. Qual Life Res. 2017;26(7):1721-1731.

49. LENT SOMA tables. Radiother Oncol. 1995;35(1):17-60.

50. Davidson SE, Trotti A, Ataman OU, Seong J, Lau FN, da Motta NW, Jeremic B. Improving the capture of adverse event data in clinical trials: the role of the International Atomic Energy Agency. Int J Radiat Oncol Biol Phys. 2007;69(4):1218-1221.

51. Ho KF, Farnell DJ, Routledge JA, Burns MP, Sykes AJ, Slevin NJ, Davidson SE. Comparison of patient-reported late treatment toxicity (LENT-SOMA) with quality of life (EORTC QLQ-C30 and QLQH\&N35) assessment after head and neck radiotherapy. Radiother Oncol. 2010;97(2):270-275.

52. Ojo B, Genden EM, Teng MS, Milbury K, Misiukiewicz KJ, Badr H. A systematic review of head and neck cancer quality of life assessment instruments. Oral Oncol. 2012;48(10):923-937.

53. Rogers SN, Ahad SA, Murphy AP. A structured review and theme analysis of papers published on "quality of life" in head and neck Cancer: 2000 to 2005. Oral Oncol. 2007;43(9):843-868.

54. Ringash J, Bezjak A. A structured review of quality of life instruments for head and neck cancer patients. Head Neck. 2001;23(3):201-213.

55. Kanatas AN, Rogers SN. A guide of the questionnaires used in the measurement of health-related quality of life in head and neck oncology. Tumori. 2008;94(5):724-731.

56. Rogers SN. Quality of life perspectives in patients with oral cancer. Oral Oncol. 2010;46(6):445-447.

57. Rogers SN, Scott B, Lowe D, Ozakinci G, Humphris GM. Fear of recurrence following head and neck cancer in the outpatient clinic. Eur Arch Otorhinolaryngol. 2010;267(12):1943-1949.

58. Rogers SN, El-Skeikha J, Lowe D. Development of a Patient Concerns Inventory (PCI) to help reveal patient concerns in the head and neck clinic. Oral Oncol. 2009;45(7):555-561.

59. Ghazali N, Lowe D, Rogers SN. Enhanced patient reported outcome measurement suitable for head and neck cancer follow-up clinics. Head Neck Oncol. 2012;4:32.

60. Rogers SN, Laher SH, Overend L, Lowe D. Importance-rating using the University of Washington quality of life questionnaire in patients treated by primary surgery for oral and oropharyngeal cancer. $J \mathrm{Cra}$ niomaxillofac Surg. 2002;30(2):125-132.

61. van der Linden N, Flach GB, de Bree R, Uyl-de Groot CA. Cost-utility of sentinel lymph node biopsy in cT1-T2N0 oral cancer. Oral Oncol. 2016;53:20-26.

62. Hutcheson KA, Lewin JS, Barringer DA, Lisec A, Gunn GB, Moore MW, Holsinger FC. Late dysphagia after radiotherapy-based treatment of head and neck cancer. Cancer. 2012;118(23):5793-5799.

63. Taibi R, Lleshi A, Barzan L, et al. Head and neck cancer survivor patients and late effects related to oncologic treatment: update of the literature. Eur Rev Med Pharmacol Sci. 2014;18(10):1473-1481.

64. Jacobson AS, Zevallos J, Smith M, et al. Quality of life after management of advanced osteoradionecrosis of the mandible. Int J Oral Maxillofac Surg. 2013;42(9):1121-1128.

65. Rogers SN, D’Souza JJ, Lowe D, Kanatas A. Longitudinal evaluation of health-related quality of life after osteoradionecrosis of the mandible. Br J Oral Maxillofac Surg. 2015;53(9):854-857.
66. Korfage A, Schoen PJ, Raghoebar GM, Roodenburg JL, Vissink A, Reintsema H. Benefits of dental implants installed during ablative tumour surgery in oral cancer patients: a prospective 5-year clinical trial. Clin Oral Implants Res. 2010;21(9):971-979.

67. Pace-Balzan A, Butterworth CJ, Dawson LJ, Lowe D, Rogers SN. The further development and validation of the Liverpool Oral Rehabilitation Questionnaire (LORQ) version 3: a cross-sectional survey of patients referred to a dental hospital for removable prostheses replacement. J Prosthet Dent. 2008;99(3):233-242.

68. Erkal EY, Canoglu D, Kaya A, et al. Assessment of early and late dysphagia using videofluoroscopy and quality of life questionnaires in patients with head and neck cancer treated with radiation therapy. Radiat Oncol. 2014;9:137.

69. Rogers SN, Thomson R, O'Toole P, Lowe D. Patients experience with long-term percutaneous endoscopic gastrostomy feeding following primary surgery for oral and oropharyngeal cancer. Oral Oncol. 2007;43(5):499-507

70. Tang Y, Luo D, Rong X, Shi X, Peng Y. Psychological disorders, cognitive dysfunction and quality of life in nasopharyngeal carcinoma patients with radiation-induced brain injury. PLoS One. 2012;7(6):e36529.

71. McLarnon C, Kulloo P, Mehanna H, Kelly C, Paleri V. Quality-of-life considerations in treatment of unresectable, recurrent head and neck cancer. Expert Rev Anticancer Ther. 2010;10(3):345-352.

72. Mesía R, Rivera F, Kawecki A, et al. Quality of life of patients receiving platinum-based chemotherapy plus cetuximab first line for recurrent and/or metastatic squamous cell carcinoma of the head and neck. Ann Oncol. 2010;21(10):1967-1973.

73. Lal P, Verma M, Kumar G, Shrivastava R, Kumar S. Initial experience of head and neck cancer patients treated in an oncologist-led palliative cancer care clinic at a tertiary cancer care center in Uttar Pradesh: is the initiatve of a full-fledged palliative care for cancer patients justified. Indian J Palliat Care. 2016;22(4):477-484.

74. Shuman AG, Yang Y, Taylor JM, Prince ME. End-of-life care among head and neck cancer patients. Otolaryngol Head Neck Surg. 2011;144(5): 733-739.

75. McMillan SC, Rodriguez C, Wang HL, Elliott A. Issues faced by family caregivers of hospice patients with head and neck cancers. ORL Head Neck Nurs. 2015;33(2):8, 10-3.

76. O'Boyle CA. The schedule for the evaluation of individual quality of life (SEIQoL). Int J Men Health. 1994;23(3):3-23.

77. Blanchard P, Volk RJ, Ringash J, Peterson SK, Hutcheson KA, Frank SJ. Assessing head and neck cancer patient preferences and expectations: a systematic review. Oral Oncol. 2016;62:44-53.

78. Kanatas AN, Rogers SN. A national survey of health-related quality of life questionnaires in head and neck oncology. Ann R Coll Surg Engl. 2004;86(1):6-10.

79. Kanatas AN, Mehanna HM, Lowe D, Rogers SN. A second national survey of health-related quality of life questionnaires in head and neck oncology. Ann R Coll Surg Engl. 2009;91(5):420-425.

80. Duman-Lubberding S, van Uden-Kraan CF, Jansen F, et al. Feasibility of an eHealth application "OncoKompas" to improve personalized survivorship cancer care. Support Care Cancer. 2016;24(5): 2163-2171.

81. Aintree University Hospitals NHS Foundation Trust. Improving Quality of Life Through the Routine Use of the Patient Concerns Inventory for Head and Neck Cancer Patients. Available from: https://clinicaltrials.gov/ct2/show/NCT03086629. NLM identifier: NCT03086629. Accessed October 9, 2017

82. Walker J, Böhnke JR, Cerny T, Strasser F. Development of symptom assessments utilising item response theory and computer-adaptive testing--a practical method based on a systematic review. Crit Rev Oncol Hematol. 2010;73(1):47-67.

83. Zigmond AS, Snaith RP. The hospital anxiety and depression scale. Acta Psychiatr Scand. 1983;67(6):361-370. 
84. Levine DW, Simmons BP, Koris MJ, Daltroy LH, Hohl GG, Fossel AH, Katz JN. A self-administered questionnaire for the assessment of severity of symptoms and functional status in carpal tunnel syndrome. J Bone Joint Surg Am. 1993;75(11):1585-1592.

85. Aitken RC. Measurement of feelings using visual analogue scales. Proc $R$ Soc Med. 1969;62(10):989-993.

86. Mohanti BK, Bansal M. Late sequelae of radiotherapy in adults. Support Care Cancer. 2005;13(10):775-780.

87. Liu YJ, Zhu GP, Guan XY. Comparison of the NCI-CTCAE version 4.0 and version 3.0 in assessing chemoradiation-induced oral mucositis for locally advanced nasopharyngeal carcinoma. Oral Oncol. 2012;48(6):554-559.

88. Epstein JB, Beaumont JL, Gwede CK, et al. Longitudinal evaluation of the oral mucositis weekly questionnaire-head and neck cancer, a patientreported outcomes questionnaire. Cancer. 2007;109(9):1914-1922.

89. Herdman M, Gudex C, Lloyd A, et al. Development and preliminary testing of the new five-level version of EQ-5D (EQ-5D-5L). Qual Life Res. 2011;20(10):1727-1736.

90. Aaronson NK, Ahmedzai S, Bergman B, et al. The European Organization for Research and Treatment of Cancer QLQ-C30: a quality-of-life instrument for use in international clinical trials in oncology. $J$ Natl Cancer Inst. 1993;85(5):365-376.

91. Hassan SJ, Weymuller EA Jr. Assessment of quality of life in head and neck cancer patients. Head Neck. 1993;15(6):485-496.

92. List MA, D'Antonio LL, Cella DF, Siston A, Mumby P, Haraf D, Vokes E. The performance status scale for head and neck cancer patients and the functional assessment of cancer therapy-head and neck scale. A study of utility and validity. Cancer. 1996;77(11):2294-2301.
93. Weymuller EA Jr, Alsarraf R, Yueh B, Deleyiannis FW, Coltrera MD. Analysis of the performance characteristics of the University of Washington Quality of Life instrument and its modification (UW-QOL-R). Arch Otolaryngol Head Neck Surg. 2001;127(5):489-493.

94. Pace-Balzan A, Cawood JI, Howell R, Lowe D, Rogers SN. The Liverpool Oral Rehabilitation Questionnaire: a pilot study. J Oral Rehabil. 2004;31(6):609-617.

95. Johnson J, Carlsson S, Johansson M, Pauli N, Rydén A, FagerbergMohlin B, Finizia C. Development and validation of the Gothenburg Trismus Questionnaire (GTQ). Oral Oncol. 2012;48(8):730-736.

96. McHorney CA, Robbins J, Lomax K, Rosenbek JC, Chignell K, Kramer AE, Bricker DE. The SWAL-QOL and SWAL-CARE outcomes tool for oropharyngeal dysphagia in adults: III. Documentation of reliability and validity. Dysphagia. 2002;17(2):97-114.

97. Beetz I, Burlage FR, Bijl HP, et al. The Groningen RadiotherapyInduced Xerostomia questionnaire: development and validation of a new questionnaire. Radiother Oncol. 2010;97(1):127-131.

98. Murphy BA, Dietrich MS, Wells N, et al. Reliability and validity of the Vanderbilt Head and Neck Symptom Survey: a tool to assess symptom burden in patients treated with chemoradiation. Head Neck. 2010;32(1):26-37.

99. Groenvold M, Petersen MA, Aaronson NK, et al. The development of the EORTC QLQ-C15-PAL: a shortened questionnaire for cancer patients in palliative care. Eur J Cancer. 2006;42(1):55-64.

100. Casarett D, Pickard A, Bailey F, et al. A nationwide VA palliative care quality measure: the family assessment of treatment at the end of life. J Palliat Med. 2008;11(1):68-75.

\section{Patient Related Outcome Measures}

\section{Publish your work in this journal}

Patient Related Outcome Measures is an international, peer-reviewed, open access journal focusing on treatment outcomes specifically relevant to patients. All aspects of patient care are addressed within the journal and practitioners from all disciplines are invited to submit their work as well as healthcare researchers and patient support groups.

\section{Dovepress}

The journal is included in PubMed. The manuscript management system is completely online and includes a very quick and fair peer-review system. Visit http://www.dovepress.com/testimonials.php to read real quotes from published authors. 